\section{MULTIVARIATE BIOLOGY}

Multivariate Statistical Analysis for Biologists By Hilary L. Seal. Pp. xi +207 . (London: Methuen and Co., Ltd., 1964.) 45s.

GATISTICAL theory applicable to records that $S$ consist of many different measurements made on each member of a sample has been widely investigated for many years. Regression and correlation analysis, introduced early in the century, were followed by generalizations of analysis of variance, canonical and principal component analysis, and many forms of factor analysis. Although all these techniques have been applied in biology, until recently the magnitude of the arithmetical tasks involved has limited their use to analyses of relatively small numbers of different characters. The computer revolution has removed this barrier, and no scientist noed now be intimidated by an analysis that involves inversion of a large matrix or determination of its eigenvalues.

This new-found freedom properly leads to an urge to explore and exploit multivariate analyses. In its train may come some excesses of enthusiasm, for example, performance of a canonical analysis when examination of each variate separately would better serve the aims of an enquiry. Until greater experience is obtained, statisticians cannot judge how far some of these techniques will prove biologically enlightening.

Dr. Seal writes for biologists, in a style that is always interesting and enthusiastic: he aims to present a wide range of multivariate methods with a minimal requirement of knowledge of other statistical theory and practice. He rightly emphasizes that a biologist should understand the logical structure of a method before subjecting his data to the appropriate computer programme. Whether he is wise to develop his theme without dependence on greater knowledge of standard statistical practice is questionable; a balanced judgmont of the role of component or factor analyses in particular biological problems is unlikely to be formed by anyone ignorant of analysis of variance and allied topics. Unfortunately, a determination to avoid dependence on standard analysis of variance makes unrealistic Dr. Seal's accounts of trivial numerical examples in his early chapters. Several analyses of measurements made at irregular intervals of time on the same plots or animals are discussed, although non-homogeneous correlations almost certainly make analysis of so few measurements useless. A reviewer who finds the analysis of variance and its ramifications the most valuable single instrument for examination of multiply-classified measurements can scarcely be sympathetic to a presentation that somewhat self-consciously avoids it and in consequence uses inappropriate models. Will any reader whose experience makes him adequate to judge share the author's preference? When he turns to multiple regression, $\mathrm{Dr}$. Seal's presentation is far more effective. He rightly stresses the importance to the biologist of easy handling of matrix operations, How surprising, then, to find him twice implying that the standard method for computing solutions of sets of linear equations is from ratios of determinants.

The second half of the book will attract greater attention as the first account of methods for the simultaneous analysis of several dependent variates directed at wide use in biology. The numerical description of a multiple linear model is particularly clear, though marred by data which fail to distinguish between measurements on different men and repeated measurements on the same man. For the reader familiar with simple matrix notation, the chapter on principal components will also be of great value. The fundamental problem of changes of scales of measurement receives comment, but scarcely sufficient discussion; the implication that difficulties disappear if all variates have been measured in the same units will not meet the needs of records including, say, height and skin thickness of men (both measured in millimetres)!
The two final chapters, on canonical and factor analyses, are more difficult. The job has been well done, but whether the time has yet come for popularizing these techniques in biology is questionable. The need for their trial under expert critical guidance is evident; despite his own thorough study, Dr. Seal finds himself unable to state the exact part they should play in the interpretation of data. However desirable a full multivariate analysis may be, undiscriminating calculations by those not able to combine familiarity with theory and appreciation of biology may be more misleading than unsophisticated analysis of single variables. It is surprising that relatively little emphasis is placed on classical discriminant analysis and its extensions, a field in which both justification and practical use are clearer than elsewhere. Less surprising is the very brief consideration of statistical taxonomy, for this subject, despite tho present great intorest, needs much development before it can be clearly expounded.

This book merits criticism on details of presentation and purpose. It should be widely studied: it is refreshingly different in plan, and it will force both biologists and statisticians to think anow about the aims of quantitative research.

D. J. Finney

\section{A PANORAMA OF GROWTH}

\section{The Growth Process in Animals}

By Dr. A. E. Noodham. Pp. xiv +522. (London: Sir Isaac Pitman and Sons, Ltd., 1964.) 70s. net.

$\mathrm{D}$

R. NEEDHAM has displayed considerable courage in undertaking the task of writing this book, which represents no less than an attempt to assess our present knowledge of the processes of growth in animals. His success in achieving this, within the limits of a book of this size, is a testimonial to the breadth of his knowledge (more than sixteen hundred references are listed) and to his ability to synthesize and correlate information from different branches of biology.

The difficulties inherent in reducing a great number of disparate facts into an orderly and mentally digestible sequence have been eased by sensible overall planning of the text. The book is divided into two parts: in the first, the actual processes involved in growth are discussed, beginning with those which are manifest in the organism as a whole, such as rate of growth and its temporal and spatial patterns, then dealing successively with aspects of growth at the diminishing levels of organs, tissues, individual cells and intracellular structures. This first section concludes with chapters on the biosynthesis of proteins and of nucloic acids, as being the most important constituents of living matter.

In the second part of the book, attention is directed to the more controversial question of the mechanisms whereby the processes of growth are controlled. Here the sequence adopted in the earlier chapters is reversed; after an initial discussion of the raw materials required, consideration is given to the controlling influence of nucleic acids and intracellular structures and to the relationship of mitosis to growth regulation. Then follow chapters on the control of growth in tissues and organs and at the systemic level, the role of genetic factors, and the effect of the environment on the organism. 'The last chapters deal with the most problematical aspects, that is, the question of the overall control of growth, and the relationships between growth and differentiation.

I do not consider myself competent to assess the accuracy of all the statements made in the book, but a few were noted as potentially misleading. Thus, on p. 96 , the definition of teratomata as "neoplasms of the gonads" would scarcely satisfy a pathologist. Again, the statement (p. 90) that it is difficult to induce growth of cells in vitro in the complete absence of embryo extract is certainly unacceptable. In the chapter on protein biosynthesis, antibody synthesis is described as occurring in plasma 